\title{
An unusual hematological presentation of gastric adenocarcinoma
}

\begin{abstract}
Introduction: Gastric malignancy remains a lethal disease, especially in developing countries. Advanced disease mostly involve peritoneum, liver, and lung. Bone involvement is rare, and bone marrow dissemination is even rarer. Hematological abnormalities may be seen as complications of gastric malignancy. Therefore knowledge of gastric malignancy possibilities spreading to bone marrow and causing hematological complications need to be taken.

Case illustration: This case report describes a 33-year-old woman who complained of weakness, waxing and waning fever, leg pain, back pain, and weight loss. The patient examination revealed pallor, and the laboratory work-up depicted severe anemia and thrombocytopenia; the peripheral blood smear was consistent with leukoerythroblastosis. Bone marrow biopsy revealed adenocarcinomas. During hospitalization, the patient presented melena, and an upper digestive endoscopy showed an ulcerated and infiltrative lesion in antrum which the histopathology reported evidence of gastric adenocarcinoma. PET-CT scan showed multiple metastatic of lymph node in neck, chest, and abdomen. Liver and bone also showed diffuse metastases.

Discussion: Our case highlights an unusual case of gastric adenocarcinoma with manifestations of bicytopenias and leukoerythroblastosis. As the diagnosis of gastric adenocarcinoma is based on endoscopic biopsy, the diagnosis can be difficult if the complaints is nonspecific, particularly in advanced stage. Diagnosis also become difficult in patients with bicytopenia and it is important to have a broad differential diagnosis when approaching a patient with bone pain. The aggressive behavior of this malignancy made poor outcome on patient.
\end{abstract}

Keywords: bicytopenia, bone marrow, gastric carcinoma, leukoerythroblastosis, PET-CT scan
Volume 9 Issue 3 - 2018

\section{Hendra Koncoro,' Eddy Setijoso,' \\ Findy Prasetiawaty,' Maisie ME Johan, ${ }^{2}$ Renaningtyas Tambun ${ }^{3}$}

'Department of Internal Medicine, Sint Carolus Hospital Jakarta, Indonesia

2Department of Radiology, Sint Carolus Hospital Jakarta, Indonesia

${ }^{3}$ Department of Pathology, Sint Carolus Hospital Jakarta, Indonesia

Correspondence: Hendra Koncoro, Department of Internal Medicine, Sint Carolus Hospital Jakarta, Indonesia,

Email hendra_koncoro@yahoo.com

Received: February 28, 2018| Published: June I2, 2018

\section{Introduction}

According to International Agency for Research on Cancer, gastric malignancy is the fifth most common cancer in the world with more than half occurred in Asia. It was responsible for 723,000 deaths worldwide in 2012. The gastric cancer still holds public health problem as Helicobacter pylori remain a burden in Asia. More than $50 \%$ of world's population reside in Asia, therefore comprehensive knowledge on this disease especially its expansion to bone marrow need to be reported. ${ }^{1,2}$

Adenocarcinoma occupied approximately ninety percent of gastric cancer histologic types, and its metastases most commonly occur in the peritoneum, liver, and lung. Gastric carcinoma rarely invade bone marrow, and only little amount of case reports show this involvement. Involvement of bone marrow by neoplasm will make impairment of bone marrow production and leading to total bone marrow aplasia. This condition is usually associated with poor prognosis.,

The case of a patient with gastric cancer and bicytopenia (anemia and thrombocytopenia) were reported. Work-up from laboratory, imaging, and histopathology showed the presence of diffuse infiltration of the bone marrow and extended metastasize to multiple organ by cells of gastric neoplasia origin. Reports regarding bone marrow metastases in gastric cancer patients in Asia were rare, therefore this case report was brought.

\section{Case report}

A 33-year-old lady presented with acute worsening of fatigue, leg pain, and back pain. She had history of irregular fever for 5 months and fatigue for 1.5 months. Other significant complaints was dyspeptic symptoms and weigh loss. She had never required red blood cell transfusions. Family history of cancer was denied. Physical examination revealed temperature $39^{\circ} \mathrm{C}$, pallor, tachycardia (124/min). Neck lymph node was palpable. A mild systolic cardiac murmur was audible in the aortic area, and the abdomen was plain, flaccid, and free of ascites and hepatomegaly. Rest of the examination was unremarkable. Her complete blood counts showed the following: erythrocytes $=2,110,000 / \mathrm{mm}^{3}$, hemoglobin $=6.0 \mathrm{mg} /$ $\mathrm{dL}$, hematocrit $=19.1 \%$, mean corpuscular volume $(\mathrm{MCV})=91 \mathrm{fL}$, and erythrocyte sedimentation rate $=27 \mathrm{~mm}$. Reticulocyte count was $2.2 \%$. The peripheral blood smear revealed the presence of polychromatic erythroblasts, metamielocytes, and mielocytes. The platelet count was initially $55 \times 109 / \mathrm{L}$, then $43 \times 109 / \mathrm{L}$ in a second measurement. Leukocytes of $13,680 / \mathrm{mm}^{3}$ showed a marked shift to the left (myelocytes 5\%, metamyelocytes $2 \%$, bands $14 \%$, segmented $61 \%$, lymphocytes $16 \%$, and monocytes $1 \%$ ). Lactic dehydrogenase determination was $2597 \mathrm{U} / \mathrm{L}$. AST was $158 \mathrm{IU} / \mathrm{L}$ and ALT was 128IU/L. Gamma GT 164U/L and alkali phosphatase 437IU/L. Renal function tests were normal. Even though blood transfusions were carried out, the hemoglobin level returned to a very low titer over the following days. Therefore, the patient was hospitalized with the 
working diagnoses of occult bleeding, myelodysplastic syndrome, and probable autoimmune disease.

Serology for hepatitis B and C, human immunodeficiency virus (HIV) and cytomegalovirus (CMV) were negative. Laboratory investigations showed positive Anti-Sm. Neck ultrasound showed lymph node enlargement (Figure 1). The abdominal computed tomography (CT) showed lymphadenopathy in the periaortic, interaortocaval portocaval, and in the lesser gastric curvature topographies.

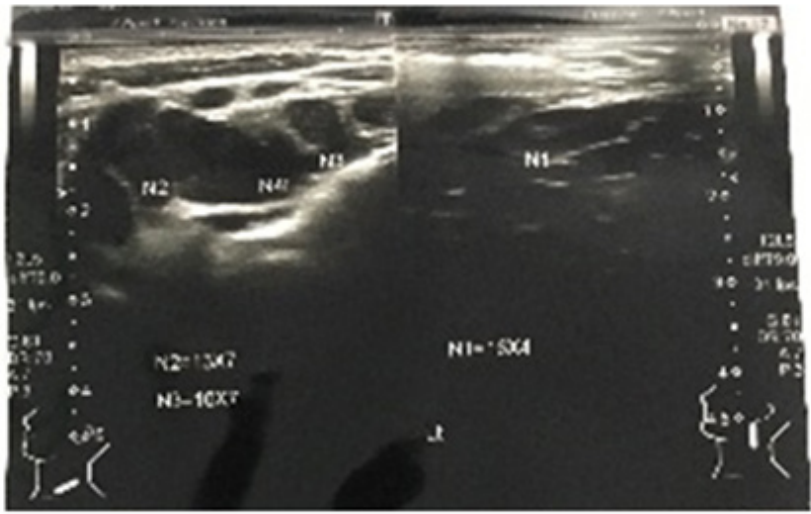

Figure I Ultrasound of neck area showed several enlargement of lymph nodes.

Because of the complete blood count and blood smear were consistent with leukoerythroblastosis, a bone marrow biopsy was performed. Bone marrow biopsy showed metastatic adenocarcinoma (Figure 2). The bone marrow was so extensively infiltrated by the neoplasia that the hematopoietic elements were scarcely represented ( $1 \%$ of the sample). The patients received two units of packed red cells and thromboapheresis.
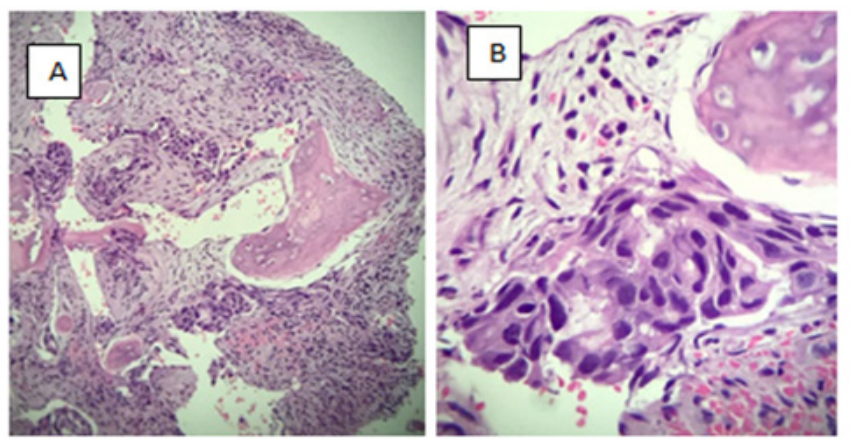

Figure 2 Pathology of the bone marrow biopsy. A-Diffuse infiltration by adenocarcinoma with mucinous background ( $\mathrm{H} \& \mathrm{E}, 200 \mathrm{x})$, B-Bone marrow biopsy revealed adenocarcinoma (H \& E, 400x).

After a suspicious episode of melena, an upper digestive endoscopy was undertaken. Gastroduodenoscopy showed growth at antrum and bile reflux erosive gastropathy (Figure 3). Biopsies were taken from the antrum. The histopathology reports showed evidence of gastric adenocarcinoma (Figure 4).

Screening for malignancy extension was performed. PET-CT scan showed multiple metastatic of hypermetabolic lymph node in bilateral supraclavicular, anterior mediastinum, paratrachea, tracheobronchial, subcarina, bilateral hilus, phrenic, coeliac, periporta, perigastrica, paraaorta, aortocava, pericava, prevertebra, mesenteric, and right parailiaca. Both hepatic lobes also showed diffuse metastase. Lytic and sclerotic lesion with destruction were seen in vertebra cervical I and II, almost all thoraco-lumbal-sacral, part of right and left costae, sternum, bilateral clavicula, bilateral scapula, bilateral humerus, pelvis, and bilateral femur suggested as bone metastases (Figure 5). Chemotherapy was planned on this case, however, one week later, patient died following acute respiratory distress syndrome due to pneumonia.
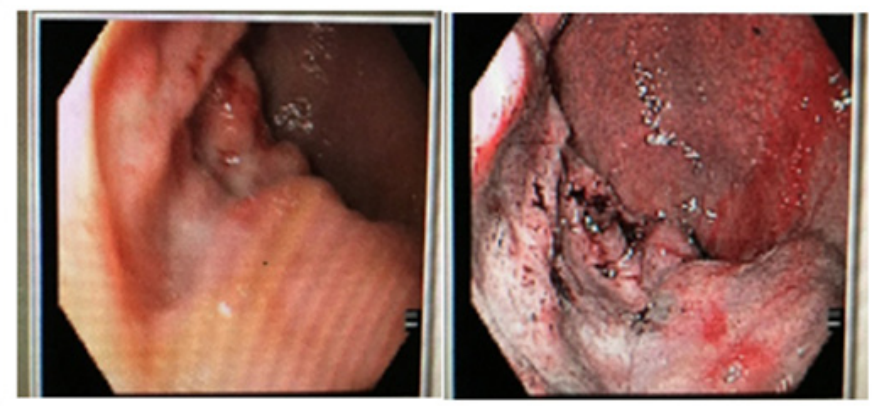

Figure 3 Esophagogastroduodenoscopy showed an ulcerated and infiltrative lesion in antrum.

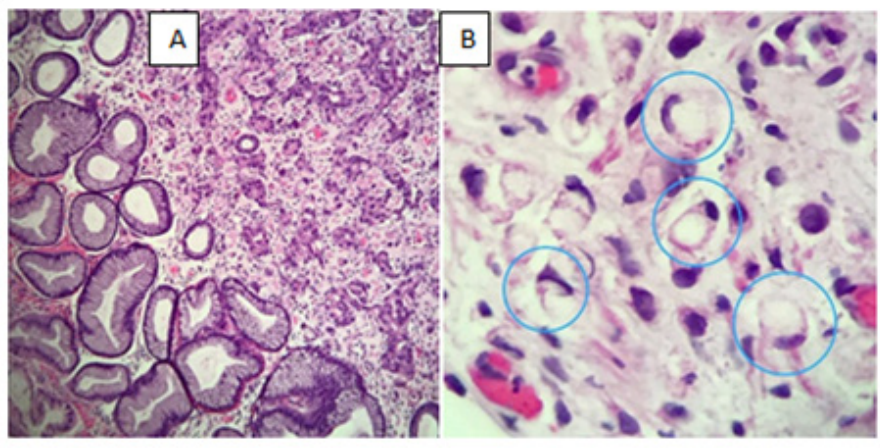

Figure 4 Pathology of the gastric biopsy. A-Lower magnification (H \& E, 400x), B-Higher magnification showed poorly differentiated adenocarcinoma with signet ring cells in circle ( $\mathrm{H} \& \mathrm{E}, 1000 \mathrm{x})$.

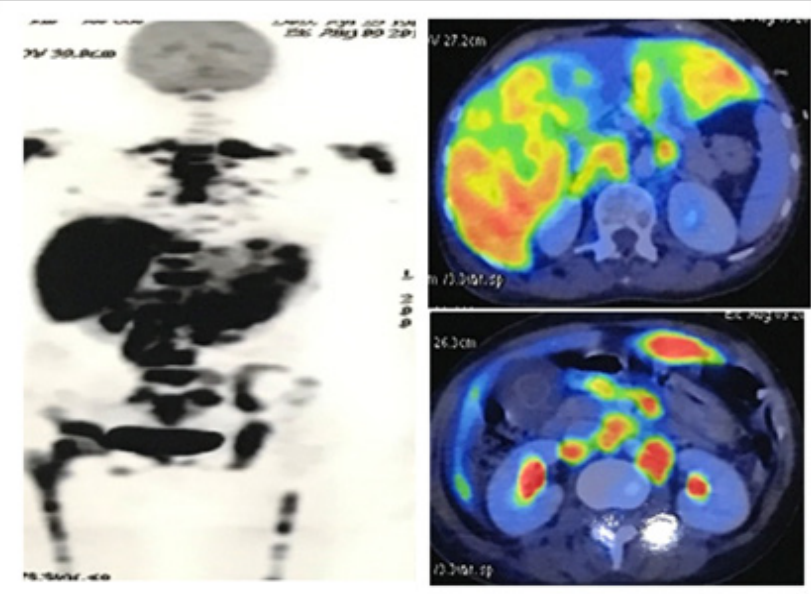

Figure 5 PET-CT scan showed multiple metastatic of lymph node in neck, chest, and abdomen. Liver and bone also showed diffuse metastases. 


\section{Discussion}

The gastric cancer is a common occurrence in developing countries. Although the incidence of gastric cancer has decreased worldwide, it is still considered as lethal malignancies worldwide. It is the second leading cause of cancer death worldwide. In Asia, gastric malignancy is the third most common cancer after breast and lung cancer. ${ }^{5}$ Gastric carcinoma usually metastasize to liver, peritoneum, and lymph nodes, and less frequently to the ovary, central nervous system, bone, lung, and soft tissue. Bone metastases are very uncommon, but when they occur the prognosis is generally poor. Bone metastasis was confirmed in approximately $10 \%$ of gastric cancer patients. ${ }^{6}$

Bone marrow infiltration is extremely rare, and occurs in a small percentage of bone metastasis. ${ }^{7}$ Biopsy studies reported by Kim et al showed that the prevalence of bone marrow infiltration was $0.024 \%$ confirmed by biopsy studies. ${ }^{8}$ Kwon et al., ${ }^{9}$ studied 26 patients with advanced gastric cancer with bone marrow involvement showing a concomitance of bone, pulmonary, and hepatic metastasis in 57\%, $11 \%$, and $3.8 \%$, respectively. The most common abnormal laboratory findings were anemia and thrombocytopenia. ${ }^{9}$ Infiltration of bone marrow is more common in patients with diffuse type gastric cancer and occurs in particularly young patients. ${ }^{4}$

The bone marrow is composed of (i) hematopoietic cells origin; (ii) mesenchymal cells origin; (iii) endothelial cells; and (iv) neural cells. Once within the blood vessels, the neoplastic cells may invade the bone marrow and actively act in this microenvironment resulting in metastatic neoplasia. Osteoclasts and the release of growth factors within the bone marrow stimulates tumor growth. ${ }^{10}$ Malignant cells of gaster induce the differentiation and activation of the osteoclasts by the production of cytokines and via the receptor activator of NFkappaB/receptor activator of NF-kappaB ligand (RANK/RANKL) system. According to Kusumoto et al.," expression of RANKL system play a critical role in pathogenesis of bone lesions. ${ }^{11}$ This case was presented with signet ring cell adenocarcinomas which showed poor prognosis adenocarcinoma.

Studies conducted in Japan on patients with gastric cancer and bone marrow infiltration have shown some particular traits: the patients are younger, there is an increased determination of alkaline phosphatase and lactate dehydrogenase, there is bone metastasis present, and a low incidence of hypercalcemia. These traits are associated with an aggressive histological patterns (poorly differentiated carcinoma) when compared with gastric cancer without bone marrow involvement. The clinical outcome of these cases worsens very quickly due to the hematopoietic suppression and hastened tumoral cells proliferation. ${ }^{11}$ This case presented with young age (33 years old), symptoms of weight loss and back pain, increased alkaline phosphatase (437U/L) and lactate dehydrogenase $(2597 \mathrm{U} / \mathrm{L})$, and leukoerythroblastosis matched types of patient with bone marrow infiltration and showed poor prognosis.

Hematological complication occurred in malignancy usually is leukoerythroblastosis. The leukoerythroblastosis refers to the presence of neutrophilia and nucleated red blood cells in the peripheral blood smear in a patient without a primary hematological disease. The bone marrow normal tissue is "pushed away" by malignant cells, caused the remaining stem cells migrate to the liver and spleen, resulting in the extramedullary hematopoiesis. The hematopoietic cells include nucleated red blood cells are prematurely released from the extramedullary sites. The refractory anemia of our patient was assumed as a result of the impaired hematopoiesis (bone marrow infiltration) and the continuous bleeding by the neoplastic gastric lesion. ${ }^{12}$ Prognosis of patients with bone marrow disseminated carcinomatosis associated with gastric cancer is poor. Chemotherapy is the only effective treatment. However, survival usually does not exceed 2 months after diagnosis is established. ${ }^{3}$

Our case highlights an unusual case of digestive malignancy with expansion to the hematology system. It seems that hematologic manifestations can be the first presentation of gastric adenocarcinoma. As the diagnosis of gastric adenocarcinoma is based mainly on an assessment of the endoscopy and biopsy, the differential diagnosis need to be taken on the basis of unknown source of bicytopenia, leukoerythroblastosis and increase in lactate dehydrogenase. This case emphasize that it is really rare that hematologic involvement occurs when malignant conditions cause bone marrow infiltration. However in this case there was a diagnostic delay that cause further bone marrow expansion in a short time. Though the diagnosis has been taken this condition usually had poor survival as seen in our case.

\section{Acknowledgements}

None.

\section{Conflict of interest}

The author declares no conflict of interest.

\section{References}

1. Ferlay J, Soerjomataram I, Dikshit R, et al. Cancer incidence and mortality worldwide: sources, methods and major patterns in GLOBOCAN 2012. Int $J$ Cancer. 2015;136(5):E359-386.

2. Rahman R, Asombang AW, Ibdah JA. Characteristics of gastric cancer in Asia. World J Gastroenterol. 2014;20(16):4483-4490.

3. Pinheiro NC, Rodrigues J, Pereira J, et al. Signet ring cell carcinoma's myelophthisis. BMJ Case Rep. 2014: bcr2014203662.

4. Dittus C, Mathew H, Malek A, et al. Bone marrow infiltration as the initial presentation of gastric signet ring cell adenocarcinoma. J Gastrointest Oncol. 2014;5(6):E113-116.

5. Rahman R, Asombang AW, Ibdah JA. Characteristics of gastric cancer in Asia. World J Gastroenterol. 2014;20(16):4483-4490.

6. Silvestris N, Pantano F, Ibrahim T, et al. Natural history of malignant bone disease in gastric cancer: final results of a multicenter bone metastasis survey. PLoS One. 2013;8(10):e74402.

7. Iguchi H. Recent aspects for disseminated carcinomatosis of the bone marrow associated with gastric cancer: What has been done for the past, and what will be needed in future? World J Gastroenterol. 2015; 21(43):12249-12260.

8. Kim HS, Yi SY, Jun HJ, et al. Clinical outcome of gastric cancer patients with bone marrow metastases. Oncology. 2007;73(3-4):192-197.

9. Kwon JY, Yun J, Kim HJ, et al. Clinical outcome of gastric cancer patients with bone marrow metastases. Cancer Res Treat. 2011;43(4):244-249.

10. Iguchi H. Recent aspects for disseminated carcinomatosis of the bone marrow associated with gastric cancer: What has been done for the past, and what will be needed in future? World $J$ Gastroenterol. 2015;21(43):12249-12260. 
11. Kusumoto H, Haraguchi M, Nozuka Y, et al. Characteristics features of disseminated carcinomatosis of the bone marrow due to gastric cancer: the pathogenesis of bone destruction. Oncol Rep. 2006;16(4):735-740.
12. Takayasu V, Goto EH, Casagrande MZ, et al. Bicytopenia and leukoerythroblastosis: a rare initial presentation of signet ring cell gastric adenocarcinoma. Autops Case Rep. 2017;7(2):55-60. 